\title{
La croyance au monde juste comme facteur explicatif de diverses réactions à des injustices professionnelles
}

\section{The belief in a just world as an explicative factor of various reactions to professional injustices}

\author{
Coralie SOUDAN $^{1}$ et Bernard GANGLOFF ${ }^{2}$ \\ ${ }^{1}$ Département de Psychologie, Université de Rouen (France). Courrier électronique : \\ soudan.coralie@orange.fr \\ ${ }^{2}$ Département de Psychologie, Université de Rouen (France). Courrier électronique : \\ bernard.gangloff@univ-rouen.fr
}

\section{Résumé}

La croyance en un monde juste a été définie comme la tendance à considérer que "les gens obtiennent ce qu'ils méritent et méritent ce qu'ils obtiennent", c'est-à-dire à considérer que le monde est, globalement, un lieu de justice. Face à un individu injustement victime d'événements négatifs et pour lequel il nous semble impossible de rétablir objectivement la justice, nous nions fréquemment l'existence de l'injustice quitte, pour cela, à responsabiliser la victime. Nous pouvons certes réagir en adoptant des stratégies de rééquilibrage passives (en responsabilisant la victime, en faisant appel à un destin compensateur,...), mais nous pouvons également adopter des stratégies plus actives susceptibles de rétablir objectivement l'injustice perçue. Nous nous sommes alors demandés si le degré de croyance au monde juste n'a pas une influence sur la stratégie de rééquilibrage choisie face à des injustices professionnelles. Nos résultats indiquent effectivement des préférences dans l'usage de telle ou telle stratégie, préférences accentuées par le genre et le statut.

\section{Abstract}

The belief in a just world is defined as the tendency to consider that "people get what they deserve and deserve what they get", i.e. to consider that the world is, globally, a place of justice. Facing an individual unjustly victim of negative events and for whom it seems impossible to restore the justice objectively, we frequently deny the existence of the unjustice, even if we say the responsible of what he gets. But if we can react by adopting "passive" reactions (using the victim's moral or behavioral responsibility, as in Lerner, or evocating a future favoring the victim,..), we can also adopt "active" strategies which can lead to an objective re-establishment of the justice. In the present study, we examined the influence of the degree of the belief in the just world on the selected strategy facing professional injustices. And effectively, our results indicate a some preferences in the use of such or strategy, preferences accentuated by the gender and the professional statute.

Mots clés : croyance en un monde juste, injustices professionnelles, réactions actives et passives, milieu organisationnel.

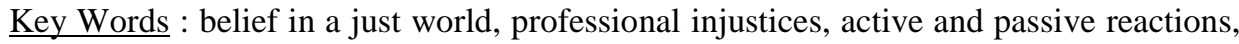
organization. 


\section{Introduction}

Les études sur la croyance en un monde juste ont notamment été initiées par des observations de Heider concernant la tendance que nous avons à mettre en relation vertu et récompense ou vice et punition (Heider, 1958, p. 235). Ces observations ont, quelques années plus tard, conduit Lerner (1965) à réaliser les premiers travaux expérimentaux sur cette croyance avec comme postulat, sous le concept de "monde ordonné", que les individus ont besoin de croire qu'ils vivent dans un monde juste, c'est-à-dire un monde dans lequel les évènements positifs arrivent aux gens bien et les évènements négatifs aux gens mauvais. La croyance en un monde juste consiste ainsi à considérer que "les gens obtiennent ce qu'ils méritent et méritent ce qu'ils obtiennent" (Lerner et Simmons, 1966, p. 204). Et il a par ailleurs été avancé que cette croyance pouvait être considérée comme relevant des normes sociales car elle fait notamment l'objet d'une valorisation sociale (Duchon et Gangloff, 2008b) et est perçue comme étant effectivement socialement valorisée (Duchon et Gangloff, 2008a ; Gangloff et Duchon, 2008).

Face à une injustice, la plupart d'entre nous éprouvent un sentiment de malaise conduisant, si la justice ne semble pas pouvoir être objectivement rétablie, à envisager des moyens permettant de la rétablir cognitivement. Les travaux de Lerner (1980), notamment, ont alors montré que, face à un individu injustement victime d'événements négatifs, nous nions fréquemment l'existence de l'injustice quitte, pour cela, à responsabiliser la victime (Lerner, 1980) ou à évoquer la malchance (Rotter, 1966) contre laquelle nous ne pouvons rien. L'individu confronté à une situation qui lui semble injuste cherche donc à maintenir ou à rétablir ses croyances en un monde juste, et arrive notamment ainsi à penser que les personnes méritent ce qui leur arrive, soit en raison de ce qu'elles ont fait (responsabilité comportementale), soit en raison de ce qu'elles sont (responsabilité morale) (Lerner, 1985/86) : s'il est possible d'attribuer le sort d'une victime à quelque chose qu'elle a ou n'a pas fait, ou si les caractéristiques personnelles de la victime justifient le sort qu'elle a subi, alors l'idée de justice est préservée. Pour autant, si certains individus restent ainsi passifs face aux injustices (en responsabilisant la victime, en faisant appel à un destin compensateur,...), d'autres décident d'adopter des stratégies plus actives susceptibles de rétablir objectivement la justice.

L'objectif du présent travail est, face à des injustices professionnelles, de faire le lien entre le degré de croyance en un monde professionnel juste et l'usage de stratégies "passives" versus "actives" de rétablissement de la justice, les premières étant essentiellement cognitives alors que les secondes permettent un rétablissemnt objectif de la justice. 


\section{Méthode}

Nous avons demandé à 171 salariés de répondre successivement à deux questionnaires : tout d'abord un questionnaire destiné à mesurer leur degré de croyance au monde juste, puis un questionnaire présentant 3 cas d'injustices professionnelles avec, à la suite de chaque cas, plusieurs réactions possibles, les unes passives et les autres traduisant des conduites actives susceptibles de rétablir objectivement la justice.

Le questionnaire de croyance en un monde juste en milieu organisationnel $^{19}$ est constitué de 36 items $^{20}$ portant sur les quatre implications de la définition du monde juste donnée par Lerner et Simmons en 1966 (actions positives récompensées, actions négatives punies, récompenses reçues méritées, et punitions reçues méritées) avec en corollaire les quatre implications injustes opposées (actions positives non récompensées,...), soit un total de huit situations ( $c f$. tableau 1). Chacune de ces huit situations était en outre déclinée dans quatre thématiques (une thématique générale au monde du travail, une deuxième centrée sur l'obtention d'un emploi, une troisième sur les possibilités de promotion et une quatrième sur les salaires); enfin, une dernière thématique (la perte d'un emploi) concernait seulement les 4 implications à essence punitive.

Les sujets devant indiquer leur accord ou désaccord à chacun des 36 items, nous avons attribué 1 point à chaque réponse attestant d'une croyance au monde juste et 0 point dans le cas inverse. Un sujet ayant systématiquement répondu positivement aux items attestant d'une croyance en un monde juste se voit donc attribué 36 points.

Tableau 1 : Les 8 dimensions utilisées

\begin{tabular}{|c|c|c|c|c|}
\hline & & & Récompense & Punition \\
\hline \multirow{2}{*}{1.} & \multirow{2}{*}{ Juste } & $\begin{array}{l}\text { Justification de la } \\
\text { sanction }\end{array}$ & $\begin{array}{l}\text { Récompense } \\
\text { méritée (RRM) }\end{array}$ & $\begin{array}{l}\text { Punition reçue } \\
\text { méritée (PRM) }\end{array}$ \\
\hline & & $\begin{array}{l}\text { Sanction } \\
\text { l'action }\end{array}$ & $\begin{array}{l}\text { Action } \quad \text { positive } \\
\text { récompensée }(\mathrm{APR})\end{array}$ & $\begin{array}{l}\text { Action négative } \\
\text { punie (ANP) }\end{array}$ \\
\hline \multirow{2}{*}{\multicolumn{2}{|c|}{ Injuste }} & $\begin{array}{l}\text { Justification de la } \\
\text { sanction }\end{array}$ & $\begin{array}{l}\text { Récompense reçue non } \\
\text { méritée (RRNM) }\end{array}$ & $\begin{array}{l}\text { Punition reçue non } \\
\text { méritée (PRNM) }\end{array}$ \\
\hline & & $\begin{array}{l}\text { Sanction } \\
\text { l'action }\end{array}$ & $\begin{array}{l}\text { Action positive non } \\
\text { récompensée (APNR) }\end{array}$ & $\begin{array}{l}\text { Action négative non } \\
\text { punie (ANNP) }\end{array}$ \\
\hline
\end{tabular}

\footnotetext{
${ }^{19}$ Nous avons choisi d'employer ce terme pour éviter toute confusion avec celui de justice organisationnelle, qui renvoie à un courant de recherche spécifique développé au départ par les travaux d'Adams en 1965.

${ }^{20}$ Sur lesquels nous avons obtenu un alpha de Cronbach de .67
} 
Le questionnaire de réactions face aux injustices professionnelles est constitué de 3 saynètes : 2 cas de népotisme se traduisant soit par une non embauche (cas 1) soit par un licenciement (cas 3), et un cas de licenciement par manque de souplesse (ou d'allégeance) de la part de la victime (cas 2).

Faisant suite à chacune des trois saynètes, neuf questions étaient présentées : la $1^{\text {ère }}$ était destinée à vérifier que la situation présentée était bien considérée comme injuste, les huit autres items comprenaient cinq propositions de conduites de passivité et trois propositions de conduites d'activisme. Les cinq conduites passives étaient : 1) la responsabilisation morale de la victime, 2) la responsabilisation comportementale de la victime, 3) l'évocation d'un futur compensant l'injustice, 4) l'évocation d'un futur favorisant la victime, et 5) l'évocation d'une impuissance historique. Quant aux conduites actives, il s'agissait d'une action de contestation collective légale, d'une action de contestation judiciaire individuelle (donc légale) et enfin d'une action de contestation individuelle mais hors normes.

Le tableau 2 synthétise la définition de chacune de ces 8 propositions réactionnelles (avec les abréviations que nous utiliserons ultérieurement).

Tableau 2 : définition de chacune des propositions réactives avec leur abréviation

\begin{tabular}{|c|c|c|}
\hline Abréviation & Dénomination & Définition \\
\hline $\mathrm{RM}$ & Responsabilité morale & $\begin{array}{l}\text { C'est la personnalité de la victime qui } \\
\text { explique ce qui lui est arrivé }\end{array}$ \\
\hline $\mathrm{RC}$ & Responsal & La victime a eu un comportement fautif \\
\hline $\mathrm{FC}$ & Futur compensateur & $\begin{array}{l}\text { Il s'agit d'une minimisation de l'aspect } \\
\text { négatif de la situation en considérant } \\
\text { celle-ci comme très éphémère et que tout } \\
\text { va bientôt rentrer dans l'ordre }\end{array}$ \\
\hline FFV & Futur favorisant la $v$ & $\begin{array}{l}\text { Il s'agit d'un renversement, par évocation } \\
\text { d'un futur positif, de l'aspect négatif de } \\
\text { la situation actuelle : la victime } \\
\text { constatera plus tard que c'est bien mieux } \\
\text { ainsi }\end{array}$ \\
\hline EIH & $\begin{array}{l}\text { Évocation d'une } \\
\text { impuissance historique }\end{array}$ & $\begin{array}{l}\text { Il est évoqué ici le fait que, dans un cas } \\
\text { comme celui-là, on est toujours } \\
\text { impuissant }\end{array}$ \\
\hline CCL & $\begin{array}{l}\text { Action de contestation } \\
\text { collective }\end{array}$ & $\begin{array}{l}\text { La victime fait appel à une association, à } \\
\text { un syndicat }\end{array}$ \\
\hline CJI & $\begin{array}{l}\text { Action de contestation } \\
\text { juridique et individuelle }\end{array}$ & $\begin{array}{l}\text { La victime entame une procédure légale } \\
\text { afin de défendre ses droits }\end{array}$ \\
\hline CIHN & $\begin{array}{l}\text { Action de contestation } \\
\text { individuelle hors norme }\end{array}$ & $\begin{array}{l}\text { La victime agit de façon contestataire } \\
\text { avec des comportements qualifiés hors } \\
\text { normes }\end{array}$ \\
\hline
\end{tabular}


A chacune des 8 propositions, les sujets devaient répondre en choisissant une réponse parmi 4 (tout à fait d'accord, d'accord, pas d'accord, pas du tout d'accord), ces 4 possibilités étant ensuite, au niveau du traitement, regroupées en $2:$ d'accord/pas d'accord. La cotation des réponses s'est faite selon le principe suivant: pour les conduites passives, il fut attribué 1 point à la réponse "d'accord" et 0 point pour "pas d'accord" ; inversement, pour les conduites actives, il fut attribué 0 point pour "d'accord" et 1 point pour la réponse "pas d'accord"

Nous obtenons ainsi : pour chaque type de conduite passive et pour l'ensemble des 3 saynètes, un indice de passivité s'échelonnant de 0 (pas du tout passif ) à 3 (totalement passif); et pour chaque type de conduite active et pour l'ensemble des 3 saynètes, un indice d'avtivisme allant de 0 (totalement actif) à 3 (pas du tout actif).

Notre échantillon de départ était constitué de 171 salariés répartis en quatre groupe selon le genre et le statut professionnel : 88 hommes (dont 48 salariés en activité et 40 chômeurs) et 83 femmes (dont 45 salariées en activité et 38 chômeuses). Mais, nous avons tout d'abord supprimé de cet échantillon tout sujet ayant considéré au moins une fois que l'un des 3 cas présentés était juste. Par ailleurs, afin d'étudier l'impact de la croyance en un monde juste, nous avons constitué 2 groupes de sujets répartis de part et d'autre de la médiane au questionnaire de croyance au monde juste (médiane = 19), ce qui signifie que nous avons aussi écarté de notre échantillon tous les sujets ayant obtenu un score situé dans la médiane. Notre échantillon final est ainsi constitué de 123 sujets dont la répartition au-dessous et audessus de la médiane est consignée dans le tableau 3. Les réactions de ces sujets a été statistiquement analysées par l'application de $t$ de Student.

Tableau 3 : répartition des sujets de part et d'autre de la médiane au questionnaire de croyance en un monde juste

\begin{tabular}{|l|c|c|c|c|c|c|}
\hline & \multicolumn{3}{|c|}{ Inf. médiane } & \multicolumn{3}{c|}{ Sup. médiane } \\
\hline & Hommes & Femmes & Total & Hommes & Femmes & Total \\
\hline $\begin{array}{l}\text { 2. Salariés } \\
(\mathbf{N}=\mathbf{6 9})\end{array}$ & 10 & 23 & 33 & 20 & 16 & 36 \\
\hline $\begin{array}{l}\text { Chômeurs (N = } \\
\text { 54) }\end{array}$ & 13 & 14 & 27 & 13 & 14 & 27 \\
\hline Total (N = 123) & 23 & 37 & 60 & 33 & 30 & 63 \\
\hline
\end{tabular}




\section{Résultats}

3.1. Résultats généraux selon le degré de croyance, le genre et le statut professionnel

Tableau 4 : réactions moyennes des sujets, quels que soient leur genre et leur statut professionnel, selon leur croyance

(les différences significatives sont indiquées en gras)

\begin{tabular}{|c|c|c|c|c|c|c|c|c|}
\hline & RM & $\overline{\mathrm{RC}}$ & $\overline{F C}$ & FFV & EIH & CCL & CJI & CIHN \\
\hline Croyants & 1.111 & 0.900 & 1.324 & 1.489 & 1.326 & 2.256 & 2.386 & 1.155 \\
\hline $\begin{array}{c}\text { Non } \\
\text { croyants }\end{array}$ & 0.882 & 0.840 & 1.063 & 1.100 & 1.558 & 2.487 & 2.310 & 1.343 \\
\hline Ecart-type & 0.718 & 0.893 & 0.947 & 1.066 & 1.036 & 0.870 & 0.856 & 0.985 \\
\hline Différence & 0.229 & 0.060 & 0.261 & 0.389 & -0.232 & 0.038 & 0.076 & -0.187 \\
\hline$T$ & 1.717 & 0.361 & 1.481 & 1.965 & -1.204 & 0.238 & 0.480 & -1.022 \\
\hline $\mathbf{P}$ & 0.044* & 0.359 & 0.071 & $0.026^{*}$ & 0.115 & 0.406 & 0.316 & 0.154 \\
\hline
\end{tabular}

D'après le tableau 4 , nous constatons que les sujets croyants (par rapport aux non croyants) expliquent davantage les injustices professionnelles par le biais des stratégies de responsabilité morale, de futur favorisant la victime (respectivement $\mathrm{p}=0.044$ et $\mathrm{p}=0.026$ ), et de manière tendantielle $(\mathrm{p}=0,071)$ par le futur compensateur.

Tableau 5 : réactions moyennes des sujets, quels que soient leur degré de croyance et leur statut professionnel, selon leur genre

(les différences significatives sont indiquées en gras)

\begin{tabular}{|c|c|c|c|c|c|c|c|c|}
\hline & RM & RC & FC & FFV & EIH & CCL & CJI & CIHN \\
\hline Hommes & 1.105 & 0.955 & 1.221 & 1.250 & 1.312 & 2.432 & 2.332 & 1.260 \\
\hline Femmes & 0.888 & 0.785 & 1.167 & 1.339 & 1.571 & 2.581 & 2.365 & 1.238 \\
\hline Ecart-type & 0.718 & 0.893 & 0.947 & 1.036 & 1.036 & 0.870 & 0.856 & 0.985 \\
\hline Différence & 0.217 & 0.170 & 0.053 & -0.089 & -0.259 & -0.149 & -0.033 & 0.022 \\
\hline T & 1.663 & 1.023 & 0.300 & -0.449 & -1.344 & -0.925 & -0.208 & 0.116 \\
\hline P & 0.054 & 0.154 & 0.382 & 0.327 & 0.091 & 0.178 & 0.418 & 0.454 \\
\hline
\end{tabular}

Au niveau du genre (tableau 5), si, d'un point vue inférentiel, nous ne pouvons conclure à aucune différence significative stricte, nous observons cependant deux tendances, les hommes ayant une moyenne plus élevée que les femmes dans l'utilisation de la responsabilité morale $(p=0,054)$, les femmes étant à l'inverse plus enclines que les hommes à évoquer une impuissance historique $(\mathrm{p}=0,091)$. 
Tableau 6 : réactions moyennes des sujets, quels que soient leur degré de croyance et leur genre, selon leur statut professionnel

(les différences significatives sont indiquées en gras)

\begin{tabular}{|c|c|c|c|c|c|c|c|c|}
\hline & RM & RC & FC & FFV & EIH & CCL & CJI & CIHN \\
\hline Salariés & 1.086 & 0.958 & 1.240 & 1.285 & 1.384 & 2.387 & 2.302 & 1.238 \\
\hline Chômeurs & 0.906 & 0.781 & 1.147 & 1.303 & 1.5 & 2.626 & 2.394 & 1.259 \\
\hline Ecart-type & 0.718 & 0.893 & 0.947 & 1.066 & 1.036 & 0.870 & 0.856 & 0.985 \\
\hline Différence & 0.180 & 0.176 & 0.093 & -0.018 & -0.116 & -0.239 & -0.092 & -0.021 \\
\hline $\mathbf{t}$ & 1.347 & 1.063 & 0.532 & -0.092 & -0.603 & -1.482 & -0.577 & -0.116 \\
\hline $\mathbf{p}$ & 0.090 & 0.145 & 0.298 & 0.463 & 0.274 & 0.070 & 0.282 & 0.454 \\
\hline
\end{tabular}

Sur le plan du statut professionnel (tableau 6), nous remarquons également deux tendances. La première se situe à nouveau parmi les stratégies passives, avec des sujets salariés qui expliquent en moyenne davantage que les chômeurs les injustices professionnelles par une responsabilité morale $(\mathrm{p}=0,090)$. Nous constatons également que les sujets salariés utilisent davantage que les chômeurs chacune des stratégies actives (notamment la contestation collective légale : $\mathrm{p}=0,070$ ).

3.2. Résultats spécifiques selon le degré de croyance, le genre et le statut professionnel

Tableau 7 : réactions moyennes des sujets hommes salariés selon leur degré de croyance (les différences significatives sont indiquées en gras)

\begin{tabular}{|c|c|c|c|c|c|c|c|c|}
\hline & RM & RC & FC & FFV & EIH & CCL & CJI & CIHN \\
\hline Croyants & 1.450 & 1.050 & 1.550 & 1.300 & 0.850 & 2.250 & 2.350 & 1.400 \\
\hline $\begin{array}{c}\text { Non } \\
\text { croyants }\end{array}$ & 1.200 & 1 & 1.100 & 0.700 & 1.400 & 2.400 & 1.900 & 1.100 \\
\hline Ecart-type & 0.721 & 0.944 & 1.033 & 0.851 & 0.944 & 1.003 & 1.060 & 0.920 \\
\hline Différence & 0.250 & 0.050 & 0.450 & 0.600 & 0.550 & 0.150 & 0.450 & 0.300 \\
\hline $\mathbf{t}$ & 1.0895 & 0.137 & 1.125 & 1.819 & 1.504 & 0.386 & 1.096 & 0.842 \\
\hline $\mathbf{p}$ & 0.189 & 0.446 & 0.135 & $\mathbf{0 . 0 4 0} *$ & 0.072 & 0.351 & 0.141 & 0.203 \\
\hline
\end{tabular}

D'après le tableau 7, la stratégie du futur favorisant la victime est significativement plus utilisée par les hommes salariés croyants que par les hommes salariés non croyants (respectivement $\mathrm{m}=1.3$ et $\mathrm{m}=0.7$ ). Il est également à souligner, malgré leur absence de signification inférentielle, que les autres stratégies passives vont systématiquement toutes dans le même sens, à savoir une plus grande utilisation par les croyants que par les non croyants, qu'il s'agisse de la responsabilité morale, de la responsabilité comportementale ou du futur compensateur. La seule exception concerne la 
stratégie de l'impuissance historique, tendantiellement plus fréquemment évoquée par les non croyants que par les croyants $(\mathrm{p}=0,072)$.

Tableau 8 : réactions moyennes des sujets hommes chômeurs selon leur degré de croyance (les différences significatives sont indiquées en gras)

\begin{tabular}{|c|c|c|c|c|c|c|c|c|}
\hline RM & RM & RC & FC & FFV & EIH & CCL & CJI & CIHN \\
\hline Croyants & 0.923 & 0.692 & 1.077 & 1.692 & 1.231 & 2.692 & 2.615 & 1.231 \\
\hline $\begin{array}{c}\text { Non } \\
\text { croyants }\end{array}$ & 0.846 & 1.077 & 1.154 & 1.308 & 1.769 & 2.385 & 2.461 & 1.308 \\
\hline Ecart-type & 0.832 & 0.951 & 0.971 & 1.217 & 0.971 & 0.760 & 0.716 & 1.022 \\
\hline Différence & 0.077 & -0.385 & -0.077 & 0.385 & -0.538 & 0.308 & 0.154 & -0.077 \\
\hline T & 0.236 & -1.031 & -0.202 & 0.806 & -1.414 & 1.033 & 0.548 & -0.192 \\
\hline P & 0.408 & 0.156 & 0.421 & 0.214 & 0.085 & 0.156 & 0.294 & 0.425 \\
\hline
\end{tabular}

On observe par contre que les hommes chômeurs croyants ne se distinguent pas des hommes chômeurs non croyants quant à leurs stratégies réactionnelles (tableau 8), à l'exception d'une tendance des non croyants à expliquer en moyenne davantage que les croyants les injustices professionnelles par l'évocation d'une impuissance historique $(\mathrm{p}=0,085)$.

Tableau 9 : réactions moyennes des sujets femmes salariées selon leur degré de croyance (les différences significatives sont indiquées en gras)

\begin{tabular}{|c|c|c|c|c|c|c|c|c|}
\hline & RM & $\mathbf{R C}$ & $\overline{F C}$ & FFV & EIH & CCL & CJI & CIHN \\
\hline Croyantes & $\overline{1}$ & $\overline{1}$ & 1.312 & 1.750 & 1.937 & 2.375 & 2.437 & 1.062 \\
\hline $\begin{array}{c}\text { Non } \\
\text { croyantes }\end{array}$ & 0.696 & 0.783 & 1 & 1.391 & 1.348 & 2.522 & 2.522 & 1.391 \\
\hline Ecart-type & 0.675 & 0.869 & 0.892 & 1.168 & 1.164 & 1.007 & 0.729 & 1.109 \\
\hline Différence & 0.304 & 0.217 & 0.313 & 0.359 & 0.590 & -0.147 & -0.084 & -0.329 \\
\hline $\mathbf{T}$ & 1.385 & 0.769 & 1.076 & 0.943 & 1.556 & -0.448 & -0.355 & 0.991 \\
\hline $\mathbf{P}$ & 0.087 & 0.223 & 0.144 & 0.176 & 0.064 & 0.382 & 0.362 & 0.164 \\
\hline
\end{tabular}

D'après le tableau 9, aucune différence significative entre les femmes salariées croyantes et non croyantes n'est à souligner quant à leurs préférences de stratégies. Pour autant, on remarque que de manière systématique chacune des stratégies passives est davantage utilisée par les croyantes que par les non croyantes (notamment l'évocation de la responsabilité morale $-\mathrm{p}=0,087$ - et de l'impuissance historique $-\mathrm{p}=0,064$ ), mais également que les non croyantes hésitent plus que les croyantes à utiliser chacune des stratégies actives. 
Tableau 10 : réactions moyennes des sujets femmes chômeuses selon leur degré de croyance (les différences significatives sont indiquées en gras)

\begin{tabular}{|c|c|c|c|c|c|c|c|c|}
\hline & RM & RC & FC & FFV & EIH & CCL & CJI & CIHN \\
\hline 3. Croyantes & 1.071 & 0.857 & 1.357 & 1.214 & 1.286 & 2.786 & 2.143 & 0.926 \\
\hline Non croyantes & 0.786 & 0.500 & 1 & 1 & 1.714 & 2.643 & 2.357 & 1.571 \\
\hline Ecart-type & 0.659 & 0.814 & 0.903 & 0.968 & 0.994 & 0.540 & 0.897 & 0.968 \\
\hline Différence & 0.286 & 0.357 & 0.357 & 0.214 & - & 0.429 & 0.143 & - \\
0.214 & -0.343 \\
\hline T & 1.147 & 1.161 & 1.046 & 0.586 & $\begin{array}{c}- \\
1.140\end{array}$ & 0.700 & $\begin{array}{c}- \\
0.632\end{array}$ & -1.757 \\
\hline P & 0.131 & 0.128 & 0.153 & 0.281 & 0.132 & 0.245 & 0.266 & $\mathbf{0 . 0 4 5}^{*}$ \\
\hline
\end{tabular}

Les femmes chômeuses croyantes (tableau 10) se positionnent significativement davantage dans la contestation individuelle hors norme par rapport aux femmes chômeuses non croyantes. En revanche, et à l'exception de l'évocation de l'impuissance historique, on observe de manière descriptive que les femmes chômeuses croyantes utilisent davantage que les non croyantes chacune des stratégies passives.

Tableau 11 : comparaisons des réactions moyennes des sujets salariés croyants selon leur genre (les différences significatives sont indiquées en gras)

\begin{tabular}{|c|c|c|c|c|c|c|c|c|}
\hline & RM & RC & FC & FFV & EIH & CCL & CJI & CIHN \\
\hline $\begin{array}{c}\text { Hommes } \\
\text { salariés } \\
\text { croyants }\end{array}$ & 1.450 & 1.050 & 1.550 & 1.300 & 0.850 & 2.250 & 2.350 & 1.400 \\
\hline $\begin{array}{c}\text { Femmes } \\
\text { salariées } \\
\text { croyantes }\end{array}$ & 1 & 1 & 1.312 & 1.750 & 1.937 & 2.375 & 2.437 & 1.062 \\
\hline Ecart-type & 0.617 & 0.857 & 1.006 & 1.017 & 0.962 & 1.157 & 0.813 & 0.966 \\
\hline Différence & 0.450 & 0.050 & 0.238 & -0.450 & -1.088 & -0.125 & -0.088 & 0.338 \\
\hline $\mathbf{t}$ & 2.174 & 0.174 & 0.704 & -1.319 & -3.369 & -0.322 & -0.321 & 1.041 \\
\hline $\mathbf{p}$ & $\mathbf{0 . 0 1 8}^{*}$ & 0.431 & 0.243 & 0.098 & $\mathbf{0 . 0 0 0}$ & 0.375 & 0.375 & 0.152 \\
\hline
\end{tabular}

Le tableau 11, intra salariés croyants, nous montre essentiellement, et de manière significative, que les hommes font plus état de la responsabilité morale et évoquent moins l'impuissance historique que les femmes. Mais nous constatons aussi une tendance des femmes à davantage utiliser le futur favorisant la victime $(\mathrm{p}=0,098)$.

Tableau 12 : comparaisons des réactions moyennes des sujets chômeurs croyants selon leur genre (les différences significatives sont indiquées en gras) 


\begin{tabular}{|c|c|c|c|c|c|c|c|c|}
\hline & RM & RC & FC & FFV & EIH & CCL & CJI & CIHN \\
\hline $\begin{array}{c}\text { Hommes } \\
\text { chômeurs } \\
\text { croyants }\end{array}$ & 0.923 & 0.692 & 1.077 & 1.692 & 1.231 & 2.692 & 2.615 & 1.231 \\
\hline $\begin{array}{c}\text { Femmes } \\
\text { chômeuses } \\
\text { croyantes }\end{array}$ & 1.071 & 0.857 & 1.357 & 1.214 & 1.286 & 2.786 & 2.143 & 0.926 \\
\hline Ecart-type & 0.845 & 0.905 & 0.803 & 1.079 & 0.963 & 0.453 & 0.769 & 0.877 \\
\hline Différence & -0.148 & -0.165 & -0.280 & 0.478 & -0.055 & -0.093 & 0.473 & 0.302 \\
\hline $\mathbf{t}$ & -0.456 & -0.473 & -0.906 & 1.150 & -0.148 & -0.536 & 1.595 & 0.894 \\
\hline $\mathbf{P}$ & 0.362 & 0.320 & 0.187 & 0.131 & 0.442 & 0.298 & 0.062 & 0.190 \\
\hline
\end{tabular}

Chez les chômeurs croyants (tableau 12), si les hommes utilisent moins que les femmes chacune des stratégies passives (à l'exception du futur favorisant la victime), les femmes font par contre tendantiellement état de plus de contestations judiciaires individuelles que les hommes $(\mathrm{p}=0,062)$.

Tableau 13 : comparaisons des réactions moyennes des sujets salariés non croyants selon leur genre (les différences significatives sont indiquées en gras)

\begin{tabular}{|c|c|c|c|c|c|c|c|c|}
\hline & RM & RC & FC & FFV & EIH & CCL & CJI & CIHN \\
\hline $\begin{array}{c}\text { Hommes } \\
\text { salariés } \\
\text { non } \\
\text { croyants }\end{array}$ & 1.200 & 1 & 1.100 & 0.700 & 1.400 & 2.400 & 1.900 & 1.100 \\
\hline $\begin{array}{c}\text { Femmes } \\
\text { salariées } \\
\text { non } \\
\text { croyantes }\end{array}$ & 0.696 & 0.783 & 1 & 1.391 & 1.348 & 2.522 & 2.522 & 1.391 \\
\hline Ecart-type & 0.772 & 0.949 & 0.896 & 1.071 & 1.186 & 0.806 & 0.961 & 0.990 \\
\hline Différence & 0.504 & 0.217 & 0.100 & -0.691 & 0.052 & -0.122 & -0.622 & -0.291 \\
\hline $\mathbf{T}$ & 1.725 & 0.605 & 0.295 & 1.704 & 0.116 & -0.399 & -1.708 & -0.777 \\
\hline $\mathbf{P}$ & $\mathbf{0 . 0 4 7}$ & 0.275 & 0.385 & $\mathbf{0 . 0 4 9}$ & 0.454 & 0.346 & $\mathbf{0 . 0 4 9}$ & 0.222 \\
\hline
\end{tabular}

Chez les salariés non croyants (tableau 13), notons tout d'abord que toutes les données obtenues vont ici pratiquement dans le même sens, avec plus d'utilisation des stratégies passives chez les hommes (sauf pour le futur favorisant la victime) mais également moins d'utilisation des stratégies actives chez les femmes. Maintenant, sur le plan des significativités, nous observons que la responsabilité morale est en moyenne plus utilisée par les hommes qui se montrent aussi plus téméraires en utilisant davantage la contestation judiciaire individuelle que les femmes ; à l'inverse, ces dernières font état de plus d'emploi du futur favorisant la victime. 
Tableau 14 : comparaisons des réactions moyennes des sujets chômeurs non croyants selon leur genre (les différences significatives sont indiquées en gras

\begin{tabular}{|c|c|c|c|c|c|c|c|c|}
\hline & RM & RC & FC & FFV & EIH & CCL & CJI & CIHN \\
\hline $\begin{array}{c}\text { Hommes } \\
\text { chômeurs } \\
\text { non } \\
\text { croyants }\end{array}$ & 0.846 & 1.077 & 1.154 & 1.308 & 1.769 & 2.385 & 2.461 & 1.308 \\
\hline $\begin{array}{c}\text { Femmes } \\
\text { chômeuses } \\
\text { non } \\
\text { croyantes }\end{array}$ & 0.786 & 0.500 & 1 & 1 & 1.714 & 2.643 & 2.357 & 1.571 \\
\hline Ecart-type & 0.634 & 0.858 & 1.052 & 1.109 & 1.003 & 0.807 & 0.859 & 1.099 \\
\hline Différence & 0.060 & 0.577 & 0.154 & 0.308 & 0.055 & -0.258 & 0.104 & -0.264 \\
\hline $\mathbf{t}$ & 0.247 & 1.745 & 0.380 & 0.720 & 0.142 & -0.831 & 0.316 & -0.623 \\
\hline $\mathbf{p}$ & 0.403 & $\mathbf{0 . 0 4 7 *}$ & 0.354 & 0.239 & 0.444 & 0.207 & 0.377 & 0.269 \\
\hline
\end{tabular}

D'après le tableau 14, nous constatons que les hommes chômeurs non croyants emploient plus la responsabilité comportementale que les femmes chômeuses non croyantes $(\mathrm{p}=0.047)$. Plus globalement, nous obtenons aussi un faisceau cohérent d'indices nous montrant systématiquement plus de réactions de passivité chez les hommes.

Tableau 15 : comparaisons des réactions moyennes des sujets hommes croyants selon leur statut professionnel

(les différences significatives sont indiquées en gras)

\begin{tabular}{|c|c|c|c|c|c|c|c|c|}
\hline & RM & RC & FC & FFV & EIH & CCL & CJI & CIHN \\
\hline $\begin{array}{c}\text { Hommes } \\
\text { salariés } \\
\text { croyants }\end{array}$ & 1.450 & 1.050 & 1.550 & 1.300 & 0.850 & 2.250 & 2.350 & 1.400 \\
\hline $\begin{array}{c}\text { Hommes } \\
\text { chômeurs } \\
\text { croyants }\end{array}$ & 0.923 & 0.692 & 1.077 & 1.692 & 1.231 & 2.692 & 2.615 & 1.231 \\
\hline Ecart-type & 0.759 & 0.911 & 1.014 & 1.062 & 0.859 & 0.925 & 0.796 & 0.935 \\
\hline Différence & 0.527 & 0.358 & 0.473 & -0.392 & -0.381 & -0.442 & -0.265 & 0.169 \\
\hline $\mathbf{T}$ & 1.948 & 1.102 & 1.310 & -1.037 & -1.245 & -1.342 & -0.936 & 0.508 \\
\hline $\mathbf{P}$ & $\mathbf{0 . 0 3 0}$ & 0.139 & 0.100 & 0.154 & 0.111 & 0.095 & 0.178 & 0.307 \\
\hline
\end{tabular}

Les hommes salariés croyants sont signiticativement plus utilisateurs de la responsabilité morale que leurs homologues chômeurs (tableau 15), et ils emploient aussi tendantiellement davantage la contestation collective légale que ne le font les chômeurs ( $\mathrm{p}=0,095)$. 
Tableau 16 : comparaisons des réactions moyennes des sujets femmes croyantes selon leur statut professionnel

(les différences significatives sont indiquées en gras)

\begin{tabular}{|c|c|c|c|c|c|c|c|c|}
\hline & RM & RC & FC & FFV & EIH & CCL & CJI & CIHN \\
\hline $\begin{array}{c}\text { Femmes } \\
\text { salariées } \\
\text { croyantes }\end{array}$ & 1 & 1 & 1.312 & 1.750 & 1.937 & 2.375 & 2.437 & 1.062 \\
\hline $\begin{array}{c}\text { Femmes } \\
\text { chômeuses } \\
\text { croyantes }\end{array}$ & 1.071 & 0.857 & 1.357 & 1.214 & 1.286 & 2.786 & 2.143 & 0.926 \\
\hline Ecart-type & 0.680 & 0.839 & 0.816 & 1.024 & 1.066 & 0.928 & 0.794 & 0.923 \\
\hline Différence & -0.071 & 0.143 & -0.045 & 0.536 & 0.652 & -0.411 & 0.295 & 0.134 \\
\hline $\mathbf{t}$ & -0.287 & 0.465 & -0.419 & 1.430 & 1.671 & -1.210 & 1.014 & 0.396 \\
\hline $\mathbf{p}$ & 0.388 & 0.323 & 0.441 & 0.082 & 0.053 & 0.118 & 0.160 & 0.347 \\
\hline
\end{tabular}

Au regard des résultats du tableau 16, nous observons deux tendances : les salariées emploient davantage le futur favorisant la victime $(p=0,082)$ et l'impuissance historique $(p=0,053)$ par rapport aux femmes chômeuses croyantes.

Tableau 17 : comparaisons des réactions moyennes des sujets hommes non croyants selon leur statut professionnel

(les différences significatives sont indiquées en gras)

\begin{tabular}{|c|c|c|c|c|c|c|c|c|}
\hline & RM & RC & FC & FFV & EIH & CCL & CJI & CIHN \\
\hline $\begin{array}{c}\text { Hommes } \\
\text { salariés } \\
\text { non } \\
\text { croyants }\end{array}$ & 1.200 & 1 & 1.100 & 0.700 & 1.400 & 2.400 & 1.900 & 1.100 \\
\hline $\begin{array}{c}\text { Hommes } \\
\text { chômeurs } \\
\text { non } \\
\text { croyants }\end{array}$ & 0.846 & 1.077 & 1.154 & 1.308 & 1.769 & 2.385 & 2.461 & 1.308 \\
\hline Ecart-type & 0.796 & 0.998 & 0.990 & 0.997 & 1.085 & 0.858 & 1.072 & 1.016 \\
\hline Différence & 0.354 & -0.077 & -0.054 & -0.608 & -0.369 & 0.015 & -0.562 & -0.208 \\
\hline $\mathbf{t}$ & 1.057 & -0.183 & -0.129 & -1.449 & -0.809 & 0.043 & -1.245 & -0.486 \\
\hline $\mathbf{p}$ & 0.151 & 0.428 & 0.449 & 0.081 & 0.214 & 0.483 & 0.113 & 0.316 \\
\hline
\end{tabular}

La comparaison des hommes salariés non croyants aux hommes chômeurs non croyants (tableau 17) ne nous montre qu'une tendance, avec des chômeurs évoquant davantage que les salariés le futur favorisant la victime $(\mathrm{p}=0,081)$. 
Tableau 18 : comparaisons des réactions moyennes des sujets femmes non croyantes selon leur statut professionnel

(les différences significatives sont indiquées en gras)

\begin{tabular}{|c|c|c|c|c|c|c|c|c|}
\hline & RM & RC & FC & FFV & EIH & CCL & CJI & CIHN \\
\hline $\begin{array}{c}\text { Femmes } \\
\text { salariées } \\
\text { non } \\
\text { croyantes }\end{array}$ & 0.696 & 0.783 & 1 & 1.391 & 1.348 & 2.522 & 2.522 & 1.391 \\
\hline $\begin{array}{c}\text { Femmes } \\
\text { chômeuses } \\
\text { non } \\
\text { croyantes }\end{array}$ & 0.786 & 0.500 & 1 & 1 & 1.714 & 2.643 & 2.357 & 1.571 \\
\hline Ecart-type & 0.660 & 0.852 & 0.956 & 1.140 & 1.122 & 0.774 & 0.810 & 1.054 \\
\hline Différence & -0.090 & 0.283 & 0 & 0.391 & -0.366 & -0.121 & 0.165 & -0.180 \\
\hline $\mathbf{T}$ & -0.403 & 0.978 & 0.000 & 1.013 & -0.963 & -0.462 & 0.600 & -0.504 \\
\hline $\mathbf{P}$ & 0.345 & 0.167 & 0.500 & 0.159 & 0.171 & 0.323 & 0.276 & 0.309 \\
\hline
\end{tabular}

D'après le tableau 18, il apparaît que les femmes salariées et chômeuses non croyantes ne se distinguent dans auncune des stratégies réactionnelles proposées.

\section{Discussion et conclusion}

Il s'avère que dans notre étude certaines stratégies de rétablissement de la justice sont préférées à d'autres, préférences effectivement fonction du degré de croyance en un monde juste. Il est ainsi mis en évidence que les croyants utilisent davantage que les non croyants aussi bien la responsabilité morale que le futur favorisant la victime ou encore (tendantiellement) le futur compensateur (tableau 5).

Nous remarquons également l'influence de nos deux variables complémentaires, c'est-à-dire le genre et le statut professionnel. Ainsi la stratégie de responsabilité morale semble préférée par les hommes (tableau 5) et notamment par les hommes salariés, qu'ils soient croyants (tableau 11) ou non croyants (tableau 13), par rapport à leurs homologues féminines. En revanche les femmes paraissent davantage se tourner vers des stratégies comme l'impuissance historique (tableau 5), notamment les femmes salariées croyantes (tableau 11) ou vers le futur favorisant la victime pour les femmes salariées non croyantes qui semblent ainsi se placer dans l'attente d'un happy end gratifiant (tableau 13). Et sur le plan du statut on observe par exemple que les salariés ont tendance à utiliser davantage que les chômeurs la responsabilité morale ou la stratégie active de contestation collective (tableau 6), notamment quant il s'agit des hommes croyants (tableau 15). 
Les résultats de cette étude exploratoire restent évidemment encore insuffisamment explicatifs. Ils nous fournissent cependant des lignes directrices nous permettant d'envisager différents lieux de poursuites. Ces différents résultats attestent en effet tout d'abord de l'existence de plusieurs types de stratégies de rétablissement cognitif de la justice : on observe ainsi que ces stratégies ne se limitent pas à celles qui sont habituellement manipulées dans les recherches sur le monde juste (essentiellement la responsabilié morale et la responsabilité comportementale). Cela ne peut donc que nous encourager à pousuivre dans cette voie en proposant d'autres stratégies de rétablissement cognitif (comme par exemple le coût trop élevé d'une contestation, indépendamment que son efficacité ; ou encore la résignation de nature transcendantale : cela arrive à tout le monde, ainsi en va-t-il de notre société). Mais cela signifie aussi que la raison pour laquelle nos variables indépendantes conduisent à davantage d'effets différenciateurs au niveau des réactions passives que des réactions actives peut provenir du nombre insuffisant de réactions actives proposées. Là encore, les études futures devront opérationnaliser et examiner l'utilisation de nouvelles réactions actives.

\section{Références}

Adams, J.S. (1965). Inequity in social exchange. In : L. Berkowitz (Ed.). Advances in experimental social psychology. New-York: Academic Press, vol. 2, 267-299.

Duchon, C. et Gangloff, B. (2008a). Clairvoyance normative de la croyance en un monde juste : une étude sur des chômeurs. Actes $d u 14^{\text {ème }}$ Congrès International de Psychologie du Travail de Langue Française. Hammamet (Tunisie). Lille : Ed. AIPTLF, vol.4, 113-122

Duchon, C. et Gangloff, B. (2008b). Valorisation de la croyance en un monde juste en situation de recrutement. 50 ème Congrès de la Société Française de Psychologie, Bordeaux.

Gangloff, B. et Duchon, C. (2008). Valorisation estimée de la croyance en une justice organisationnelle. $15^{\text {ème }}$ Congrès de l'AIPTLF, Québec (Canada).

Heider F. (1958). The psychology of interpersonal relations. New-York : Wiley. Lerner, M.J. (1965). Evaluation of performance as a function of performer's reward and attractiveness. Journal of Personality and Social Psychology, 1, 355-360.

Lerner, M. J. (1980). The belief in a just world: A fundamental delusion. New York, Plenum Press.

Lerner, M. J. (1985-86). La théorie de la justice ou le besoin de se justifier. Bulletin de Psychologie, 39, 205-211.

Lerner, M.J. et Simmons, C.H. (1966). Observer's reaction to the "innocent victim": compassion or rejection? Journal of Personality and Social Psychology, 4, 203210.

Rotter, J.B. (1966). Generalized expectancies for internal versus external control of reinforcement. Psychological Monographs, 80 (609), 1-28. 